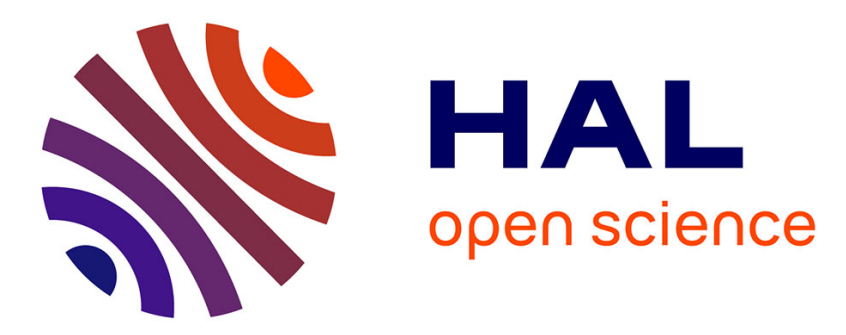

\title{
Finite time observation of nonlinear time-delay systems with unknown inputs
}

Gang Zheng, Jean-Pierre Barbot, Driss Boutat, Thierry Floquet, Jean-Pierre Richard

\section{- To cite this version:}

Gang Zheng, Jean-Pierre Barbot, Driss Boutat, Thierry Floquet, Jean-Pierre Richard. Finite time observation of nonlinear time-delay systems with unknown inputs. NOLCOS2010, 2010, Bologna, Italy. inria-00467966

\section{HAL Id: inria-00467966 \\ https://hal.inria.fr/inria-00467966}

Submitted on 29 Mar 2010

HAL is a multi-disciplinary open access archive for the deposit and dissemination of scientific research documents, whether they are published or not. The documents may come from teaching and research institutions in France or abroad, or from public or private research centers.
L'archive ouverte pluridisciplinaire HAL, est destinée au dépôt et à la diffusion de documents scientifiques de niveau recherche, publiés ou non, émanant des établissements d'enseignement et de recherche français ou étrangers, des laboratoires publics ou privés. 


\title{
Finite time observation of nonlinear time-delay systems with unknown inputs
}

\author{
G. Zheng* J.-P. Barbot, ${ }^{* *}$ D. Boutat ${ }^{* * *}$ T. Floquet ${ }^{* * * *}$ \\ J.-P. Richard ${ }^{* * * * *}$ \\ * INRIA Lille-Nord Europe, 40 Avenue Halley, 59650 Villeneuve \\ d'Ascq, France \\ ** ECS, ENSEA, 6 Av. du Ponceau, 95014 Cergy, France \\ *** ENSI de Bourges, Insitut PRISME, 88 Boulevard de Lahitolle, \\ 18020 Bourges, France \\ **** LAGIS UMR CNRS 8146, Ecole Centrale de Lille, BP 48, 59651 \\ Villeneuve d'Ascq, France
}

\begin{abstract}
Causal and non-causal observability are discussed in this paper for nonlinear timedelay systems. By extending the Lie derivative for time-delay systems in the algebraic framework introduced by Xia et al. (2002), we present a canonical form and give sufficient condition in order to deal with causal and non-causal observations of state and unknown inputs of time-delay systems.
\end{abstract}

Keywords: Time-delay systems, Observability, Causality, Canonical form

\section{INTRODUCTION}

Observation or estimation is one of the most important problems in control theory. For nonlinear systems without delays, the observability problem has been exhaustively studied, and is characterized respectively by Hermann and Krener (1977); Sontag (1984); Krener (1985) from a differential point of view, and by Diop and Fliess (1991) from an algebraic point of view. For observable systems, many types of nonlinear observers were proposed, such as high-gain observer in Gauthier et al. (1992), algebraic observer in Barbot et al. (2007), sliding mode observer in Xiong and Saif (2001); Floquet and Barbot (2007) and so on.

However, unlike nonlinear systems without delays, the analysis of properties for time-delay system is more complicated (see the surveys in Sename and Briat (2001) and Richard (2003)). For linear time-delay systems, various aspects of the observability problem have been studied in the literature, by different methods such as functional analytic approach (Bhat and Koivo (1976)), algebraic approach (Brewer et al. (1986); Sontag (1976); Fliess and Mounier (1998)), and so on (Przyiuski and Sosnowski (1984)). The theory of non-commutative rings has been applied to analyze nonlinear time-delay systems firstly by Moog et al. (2000) for the disturbance decoupling problem of nonlinear time-delay system, and for observability of nonlinear timedelay systems with known inputs by Xia et al. (2002), for identifiability of parameter for nonlinear time-delay systems in Zhang et al. (2006), and for state elimination and delay identification of nonlinear time-delay systems by Anguelova and Wennberga (2008). In this algebraic framework, the left Ore ring of non-commutative polynomials defined over the field of meromorphic functions is used for the analysis of nonlinear time-delay systems, since the rank of a module over this ring is well defined and can be used to characterize controllability, observability and identifiability of nonlinear time-delay systems.

Concerning observer design for linear and nonlinear timedelay systems, see Boutayeb (2001); Pepe (2001); Germani et al. (2002); Darouach (2006); Seuret et al. (2007); Sename and Briat (2007); Ibrir (2009) and the references therein.

In this paper, based on the algebraic framework proposed by Xia et al. (2002), we give a general definition of observability for time-delay systems with unknown inputs. Then we generalize the notation of the Lie derivative for nonlinear systems with time-delay, and redefine the relative degree and observability indices for nonlinear time-delay systems by using the theory of non-commutative rings. After that a canonical form is derived and sufficient conditions are given to treat causal and non-causal observations of states and unknown inputs of time-delay systems.

\section{ALGEBRAIC FRAMEWORK}

Denote $\tau$ the basic time delay, and assume that the times delays are multiple times of $\tau$. Consider the following nonlinear time-delay system:

$$
\left\{\begin{array}{l}
\dot{x}=f(x(t-i \tau))+\sum_{j=0}^{s} g^{j}(x(t-i \tau)) u(t-j \tau) \\
y=h(x(t-i \tau))=\left[h_{1}(x(t-i \tau)), \ldots, h_{p}(x(t-i \tau))\right]^{T} \\
x(t)=\psi(t), u(t)=\varphi(t), t \in[-s \tau, 0]
\end{array}\right.
$$

where $x \in W \subset R^{n}$ denotes the state variables, $u=$ $\left[u_{1}, \ldots, u_{m}\right]^{T} \in R^{m}$ is the unknown admissible input, $y \in$ $R^{p}$ is the measurable output. Without loss of generality, we assume that $p \geq m$. And $i \in S_{-}=\{0,1, \ldots, s\}$ is a finite set of constant time-delays, $f, g^{j}$ and $h$ are meromorphic 
functions $^{1}, f(x(t-i \tau))=f(x, x(t-\tau), \ldots, x(t-s \tau))$ and $\psi:[-s \tau, 0] \rightarrow R^{n}$ and $\varphi:[-s \tau, 0] \rightarrow R^{m}$ denote unknown continuous functions of initial conditions. In this work, it is assumed that (1) with $u=0$ is locally observable, and for initial conditions $\psi$ and $\varphi$, (1) admits a unique solution.

Based on the algebraic framework introduced in Xia et al. (2002), let $\mathcal{K}$ be the field of meromorphic functions of a finite number of the variables from $\left\{x_{j}(t-i \tau), j \in[1, n], i \in\right.$ $\left.S_{-}\right\}$. With the standard differential operator $d$, define the vector space $\mathcal{E}$ over $\mathcal{K}$ :

$$
\mathcal{E}=\operatorname{span}_{\mathcal{K}}\{d \xi: \xi \in \mathcal{K}\}
$$

which is the set of linear combinations of a finite number of one-forms from $d x_{j}(t-i \tau)$ with row vector coefficients in $\mathcal{K}$. For the sake of simplicity, we introduce backward time-shift operator $\delta$, which means

$$
\delta^{i} \xi(t)=\xi(t-i \tau), \xi(t) \in \mathcal{K}, \text { for } i \in Z^{+}
$$

and

$$
\begin{aligned}
\delta^{i}(a(t) d \xi(t)) & =\delta^{i} a(t) \delta^{i} d \xi(t) \\
& =a(t-i \tau) d \xi(t-i \tau)
\end{aligned}
$$

for $a(t) d \xi(t) \in \mathcal{E}$, and $i \in Z^{+}$.

Let $\mathcal{K}(\delta]$ denote the set of polynomials of the form

$$
a(\delta]=a_{0}(t)+a_{1}(t) \delta+\cdots+a_{r_{a}}(t) \delta^{r_{a}}
$$

where $a_{i}(t) \in \mathcal{K}$. The addition in $\mathcal{K}(\delta]$ is defined as usual, but the multiplication is given as

$$
a(\delta] b(\delta]=\sum_{k=0}^{r_{a}+r_{b}} \sum_{i+j=k}^{i \leq r_{a}, j \leq r_{b}} a_{i}(t) b_{j}(t-i \tau) \delta^{k}
$$

Note that $\mathcal{K}(\delta]$ satisfies the associative law and it is a noncommutative ring (see Xia et al. (2002)). However, it is proved that the ring $\mathcal{K}(\delta]$ is a left Ore ring (Ježek (1996); Xia et al. (2002)), which enables to define the rank of a module over this ring. Let $\mathcal{M}$ denote the left module over $\mathcal{K}(\delta]$

$$
\mathcal{M}=\operatorname{span}_{\mathcal{K}(\delta]}\{d \xi, \xi \in \mathcal{K}\}
$$

where $\mathcal{K}(\delta]$ acts on $d \xi$ according to (2) and (3).

With the definition of $\mathcal{K}(\delta],(1)$ can be rewritten in a more compact form as follows:

$$
\begin{cases}\dot{x} & =f(x, \delta)+\sum_{i=1}^{m} G_{i} u_{i}(t) \\ y & =h(x, \delta) \\ x(t) & =\psi(t), u(t)=\varphi(t), t \in[-s \tau, 0]\end{cases}
$$

where $f(x, \delta)=f(x(t-i \tau))$ and $h(x, \delta)=h(x(t-i \tau))$ with entries belonging to $\mathcal{K}, G_{i}=\sum_{j=0}^{s} g_{i}^{j} \delta^{j}$ with entries belonging to $\mathcal{K}(\delta]$. It is assumed that $\operatorname{rank}_{\mathcal{K}(\delta]} \frac{\partial h}{\partial x}=p$, which implies that $\left[h_{1}, \ldots, h_{p}\right]^{T}$ are independent functions of $x$ and its backward shifts.

\section{NOTATIONS AND DEFINITIONS}

Similar to observability definitions for nonlinear systems without delays given in Hermann and Krener (1977) and in Diop and Fliess (1991), a definition of observability for time-delay systems is given in Marquez-Martinez et al. (2002). A more generic definition is stated here as follows:

\footnotetext{
1 means quotients of convergent power series with real coefficients
} Conte et al. (1999); Xia et al. (2002).
Definition 1. System (1) is locally observable if the state $x(t) \in W \subset R^{n}$ can be expressed as a function of the output and its derivatives with their backward and forward shifts as follows:

$$
x(t)=\alpha\left(y(t-j \tau), \ldots, y^{(k)}(t-j \tau)\right)
$$

for $j \in Z$ and $k \in Z^{+}$.

Remark 1. Different to the definition in Marquez-Martinez et al. (2002) where only forward shifts are involved in (7), Definition 1 is more generic and covers the following cases:

(1) Assume (7) is satisfied for $j=0$ and $k \in Z^{+}$. This means that no time-delay is involved in (7) and Definition 1 is equivalent to the one for nonlinear systems without delays.

(2) Assume (7) is satisfied for $j \in Z^{+}$and $k \in Z^{+}$. This implies that only backward shifts can be found in (7), then $x(t) \in W$ of (1) depends only on the past values of the output and their derivatives. So the observability is causal.

(3) Assume (7) is satisfied for $j \in Z$ and $k \in Z^{+}$. This means that both backward and forward shifts appear in (7). So $x(t) \in W$ is a function of both the past and future values of the output and their derivatives, which implies that the observability of (1) is not causal.

Following the same principle of Definition 1, we make the following definition for the unknown inputs.

Definition 2. The unknown input $u(t)$ can be estimated if it can be written as a function of the derivatives of the output and its derivatives with backward and forward shifts, i.e.

$$
u(t)=\beta\left(y(t-j \tau), \ldots, y^{(k)}(t-j \tau)\right)
$$

for $j \in Z$ and $k \in Z^{+}$.

The following example illustrates Definition 1 and 2 .

Example 1. Consider the following system

$$
\left\{\begin{array}{l}
\dot{x}_{1}=x_{2}+\delta x_{1} \\
\dot{x}_{2}=\delta^{2} x_{2}-\delta x_{3} \\
\dot{x}_{3}=\delta x_{4}+\delta u_{1}+\delta^{4} u_{2} \\
\dot{x}_{4}=\delta u_{2} \\
y_{1}=x_{1} \\
y_{2}=\delta x_{4}
\end{array}\right.
$$

A straightforward calculation gives

$$
\left\{\begin{array}{l}
x_{1}(t)=y_{1}(t) \\
x_{2}(t)=\dot{y}_{1}(t)-y_{1}(t-\tau) \\
x_{3}(t)=\dot{y}_{1}(t-\tau)-y_{1}(t-2 \tau)-\ddot{y}_{1}(t+\tau)+\dot{y}_{1}(t) \\
x_{4}(t)=y_{2}(t+\tau)
\end{array}\right.
$$

and

$$
\left\{\begin{aligned}
u_{1}(t) & =\ddot{y}_{1}(t)-\dot{y}_{1}(t-\tau)-\dddot{y}_{1}(t+2 \tau)+\ddot{y}_{1}(t+\tau) \\
& -y_{2}(t+\tau)-\dot{y}_{2}(t-\tau) \\
u_{2}(t) & =\dot{y}(t+2 \tau)
\end{aligned}\right.
$$

It can be seen that $x_{1}$ and $x_{2}$ are causally observable since they depend only on the outputs and their past values. However $x_{3}, x_{4}, u_{1}$ and $u_{2}$ are non causally observable since they also depend on the future values of the outputs. So according to Definition 1 and 2, system (8) is observable, but the observability is not causal.

Definition 3. (Unimodular matrix) [Marquez-Martinez et al. (2002)] Matrix $A \in \mathcal{K}^{n \times n}(\delta]$ is said to be unimodular over 
$\mathcal{K}(\delta]$ if it has a left inverse $A^{-1} \in \mathcal{K}^{n \times n}(\delta]$, such that $A^{-1} A=I_{n \times n}$.

Remark 2. An algorithm to check whether a matrix of $\mathcal{K}^{n \times n}(\delta]$ is unimodular is stated in Marquez-Martinez et al. (2002).

Definition 4. (Change of coordinate) [Marquez-Martinez et al. (2002)] For system (1), $z=\phi(\delta, x) \in \mathcal{K}^{n \times 1}$ is a causal change of coordinate over $\mathcal{K}$ for $(1)$ if there exist locally a function $\phi^{-1} \in \mathcal{K}^{n \times 1}$ and some constants $c_{1}, \cdots, c_{n} \in N$ such that

$$
\operatorname{diag}\left\{\delta^{c_{i}}\right\} x=\phi^{-1}(\delta, z) .
$$

The change of coordinate is bicausal over $\mathcal{K}$ if $\max \left\{c_{i}\right\}=$ 0 , i.e. $x=\phi^{-1}(\delta, z)$.

Note that the relative degree for nonlinear systems without delays is well defined via the Lie derivative (see Isidori (1995)), then many efforts have been done to extend the classical Lie derivative for nonlinear time-delay systems. In Germani et al. (2001, 2002), authors defined the socalled delay relative degree for a class of nonlinear timedelay systems with only single delay, by augmenting the dimension of the studied system. In Oguchi et al. (2002); Oguchi and Richard (2006), authors introduced the delayed state derivative and delayed state bracket, which are the extensions of the conventional Lie derivative. However, those definitions are still built on the theory of commutative rings, which makes the analysis of observability for nonlinear time-delays systems still complicated. Hence in what follows we try to characterize the relative degree and observability indices for nonlinear time-delay systems by extending the Lie derivative in the algebraic framework of Xia et al. (2002), from non-commutative rings point of view.

Let $f(x(t-j \tau))$ and $h(x(t-j \tau))$ for $0 \leq j \leq s$ respectively be an $n$ and $p$ dimensional vector with entries $f_{r} \in \mathcal{K}$ for $1 \leq r \leq n$ and $h_{i} \in \mathcal{K}$ for $1 \leq i \leq p$. Let

$$
\frac{\partial h_{i}}{\partial x}=\left[\frac{\partial h_{i}}{\partial x_{1}}, \cdots, \frac{\partial h_{i}}{\partial x_{n}}\right] \in \mathcal{K}^{1 \times n}(\delta]
$$

where for $1 \leq r \leq n$ :

$$
\frac{\partial h_{i}}{\partial x_{r}}=\sum_{j=0}^{s} \frac{\partial h_{i}}{\partial x_{r}(t-j \tau)} \delta^{j} \in \mathcal{K}(\delta]
$$

then the Lie derivative for nonlinear systems without delays can be extended to nonlinear time-delay systems in the framework of Xia et al. (2002) as follows

$$
L_{f} h_{i}=\frac{\partial h_{i}}{\partial x}(f)=\sum_{r=1}^{n} \sum_{j=0}^{s} \frac{\partial h_{i}}{\partial x_{r}(t-j \tau)} \delta^{j}\left(f_{r}\right) \in \mathcal{K}
$$

For $j=0,(10)$ is the classical definition of the Lie derivative of $h$ along $f$. For $h_{i} \in \mathcal{K}$, define

$$
L_{G_{i}} h_{i}=\frac{\partial h_{i}}{\partial x}\left(G_{i}\right) \in \mathcal{K}(\delta]
$$

By the above definition of Lie derivative, we can define the relative degree in the following way:

Definition 5. (Relative degree) System (6) has relative degree $\left(\nu_{1}, \cdots, \nu_{p}\right)$ in an open set $W \subseteq R^{n}$ if, for $1 \leq i \leq p$, the following conditions are satisfied :

(1) for all $x \in W, L_{G_{j}} L_{f}^{r} h_{i}=0$, for all $1 \leq j \leq m$ and $0 \leq r<\nu_{i}-1$
(2) there exists $x \in W$ such that $\exists j \in[1, m]$, $L_{G_{j}} L_{f}^{\nu_{i}-1} h_{i} \neq 0$.

If for $1 \leq i \leq p,(1)$ is satisfied for all $r \geq 0$, then we set $\nu_{i}=\infty$.

Remark 3. This definition of relative degree for time-delay system is equivalent to the definition given in Moog et al. (2000).

Since (6) is locally observable when $u=0$, then one can define, for (6), the so-called observability indices introduced in Krener (1985). Let

$$
\mathcal{F}_{k}:=\operatorname{span}_{\mathcal{K}(\delta]}\left\{d h, d L_{f} h, \cdots, d L_{f}^{k-1} h\right\}
$$

for $1 \leq k \leq n$. And it was shown that the filtration of $\mathcal{K}(\delta]$-module satisfies

$$
\mathcal{F}_{1} \subset \mathcal{F}_{2} \subset \cdots \subset \mathcal{F}_{n}
$$

then we define

$$
\begin{aligned}
& d_{1}=\operatorname{rank}_{\mathcal{K}(\delta]} \mathcal{F}_{1} \\
& d_{k}=\operatorname{rank}_{\mathcal{K}(\delta]} \mathcal{F}_{k}-\operatorname{rank}_{\mathcal{K}(\delta]} \mathcal{F}_{k-1}
\end{aligned}
$$

for $2 \leq k \leq n$. Let

$$
k_{i}=\operatorname{card}\left\{d_{k} \geq i, 1 \leq k \leq n\right\}
$$

then $\left(k_{1}, \cdots, k_{p}\right)$ are the observability indices, and $\sum_{i=1}^{p} k_{i}=$ $n$ since it is assumed that (6) is observable with $u=0$. Reordering, if necessary, the output components of (6), such that

$$
\begin{aligned}
& \operatorname{rank}_{\mathcal{K}(\delta]} \frac{\partial\left[h, L_{f} h, \cdots, L_{f}^{n-1} h\right]^{T}}{\partial x} \\
& =\operatorname{rank}_{\mathcal{K}(\delta]} \frac{\partial\left[h_{1}, L_{f} h_{1}, \cdots, L_{f}^{k_{1}-1} h_{1}, \cdots, h_{p}, L_{f} h_{p}, \cdots, L_{f}^{k_{p}-1} h_{p}\right]^{T}}{\partial x} \\
& =k_{1}+\cdots+k_{p}=n
\end{aligned}
$$

Since $\operatorname{rank}_{\mathcal{K}(\delta]} \frac{\partial h}{\partial x}=p$, then observability indices $\left(k_{1}, \cdots, k_{p}\right)$ for $\left(h_{1}, \cdots, h_{p}\right)$ are well defined, but the order may be not unique.

\section{CANONICAL FORM AND CAUSAL OBSERVABILITY}

After having defined the relative degree and observability indices via the extended Lie derivative for nonlinear timedelay systems in the framework of non-commutative rings, we are ready to state the following theorem.

Theorem 1. For $1 \leq i \leq p$, denote $k_{i}$ the observability indices and $\nu_{i}$ the relative degree index for $y_{i}$ of (6), and note $\rho_{i}=\min \left\{\nu_{i}, k_{i}\right\}$. Then there exists a change of coordinate $\phi(x, \delta) \in \mathcal{K}^{n \times 1}$, such that (6) can be transformed into the following form:

$$
\begin{aligned}
\dot{z}_{i} & =A_{i} \dot{z}_{i}+B_{i} V_{i} \\
\dot{\xi} & =\alpha(z, \xi, \delta)+\beta(z, \xi, \delta) u \\
y_{i} & =C_{i} z_{i}
\end{aligned}
$$

where 


$$
\begin{aligned}
z_{i} & =\left(h_{i}, \cdots, L_{f}^{\rho_{i}-1} h_{i}\right)^{T} \in \mathcal{K}^{\rho_{i} \times 1} \\
A_{i} & =\left(\begin{array}{cccc}
0 & 1 & \cdots & 0 \\
\vdots & \vdots & \ddots & \vdots \\
0 & 0 & \cdots & 1 \\
0 & 0 & \cdots & 0
\end{array}\right) \in R^{\rho_{i} \times \rho_{i}}, B_{i}=\left(\begin{array}{c}
0 \\
\vdots \\
0 \\
1
\end{array}\right) \in R^{\rho_{i} \times 1}, \\
V_{i} & =L_{f}^{\rho_{i}} h_{i}(x, \delta)+\sum_{j=1}^{m} L_{G_{j}} L_{f}^{\rho_{i}-1} h_{i}(x, \delta) u_{j} \in \mathcal{K} \\
\alpha & \in \mathcal{K}^{l \times 1}, \beta \in \mathcal{K}^{l \times 1}(\delta] \text { with } l=n-\sum_{j=1}^{p} \rho_{j} \\
C_{i} & =(1,0, \cdots, 0) \in R^{1 \times \rho_{i}}
\end{aligned}
$$

Moreover if $k_{i}<\nu_{i}$, one has $V_{i}=L_{f}^{\rho_{i}} h_{i}=L_{f}^{k_{i}} h_{i}$.

Proof 1. According to the definition of $k_{i}$, for $1 \leq i \leq p$, one has

$$
\operatorname{rank}_{\mathcal{K}(\delta]} \frac{\partial\left[h_{i}, L_{f} h_{i}, \cdots, L_{f}^{k_{i}-1} h_{i}\right]^{T}}{\partial x}=k_{i}
$$

since $\rho_{i}=\min \left\{k_{i}, \nu_{i}\right\}$, one gets

$$
\operatorname{rank}_{\mathcal{K}(\delta]} \frac{\partial\left[h_{i}, L_{f} h_{i}, \cdots, L_{f}^{\rho_{i}-1} h_{i}\right]^{T}}{\partial x}=\rho_{i}
$$

which implies that the components of

$$
z_{i}=\left(h_{i}, \cdots, L_{f}^{\rho_{i}-1} h_{i}\right)^{T}
$$

are linearly independent over $\mathcal{K}(\delta]$. Denote

$$
z=\left(z_{1}^{T}, \cdots, z_{p}^{T}\right)^{T} \in \mathcal{K}^{n-l}, \text { with } l=\sum_{j=1}^{p} \rho_{j},
$$

and choose $l$ variables $\xi \in \mathcal{K}^{l}$, such that $\left(z^{T}, \xi^{T}\right)^{T}=$ $\phi(x, \delta) \in \mathcal{K}^{n}$ is a well-defined change of coordinate. Then for $1 \leq i \leq p$ and $1 \leq j \leq \rho_{i}-1$, one has

$$
\begin{aligned}
\dot{z}_{i, j} & =z_{i, j+1} \\
\dot{z}_{i, \rho_{i}} & =L_{f}^{\rho_{i}} h_{i}+\sum_{j=1}^{m} L_{G_{j}} L_{f}^{\rho_{i}-1} h_{i}(x, \delta) u_{j} \\
y_{i} & =z_{i, 1}=C_{i} z_{i}
\end{aligned}
$$

and

$$
\dot{\xi}=\alpha(z, \xi, \delta)+\beta(z, \xi, \delta) u
$$

which means (6) can be transformed into form (11-13).

Moreover, according to the definition of $\nu_{i}$, for all $1 \leq j \leq$ $m$ and $0 \leq r<\nu_{i}-1$, one has

$$
\begin{aligned}
L_{G_{j}} L_{f}^{r} h_{i} & =\frac{\partial\left(L_{f}^{r} h_{i}\right)}{\partial x}\left(G_{j}\right)=0 \\
L_{G_{j}} L_{f}^{\nu_{i}-1} h_{i} & =\frac{\partial\left(L_{f}^{v_{i}} h_{i}\right)}{\partial x}\left(G_{j}\right) \neq 0
\end{aligned}
$$

Hence, if $k_{i}<\nu_{i}$, then $\rho_{i}=k_{i}$ and one has $L_{G_{j}} L_{f}^{k_{i}-1} h_{i}(x, \delta)=$ Concerning the reconstruction of unknown inputs, rewrite $L_{G_{j}} L_{f}^{\rho_{i}-1} h_{i}(x, \delta)=0$, which yields $V_{i}=L_{f}^{\rho_{i}} h_{i}=L_{f}^{k_{i}} h_{i}$.

Remark 4. For (11-13), one can find observers in the literature (Xiong and Saif (2001); Floquet and Barbot (2007)) such that $z_{i}$ for $1 \leq i \leq p$ can be estimated in finite time due to the triangular structure. In addition, one can also obtain the information $V_{i}=y_{i}^{\left(\rho_{i}\right)}$ in finite time.

For (11), note

$$
H(x, \delta)=\Psi(x, \delta)+\Gamma(x, \delta) u
$$

with

$$
H(x, \delta)=\left(\begin{array}{c}
h_{1}^{\left(\rho_{1}\right)} \\
\vdots \\
h_{p}^{\left(\rho_{p}\right)}
\end{array}\right), \Psi(x, \delta)=\left(\begin{array}{c}
L_{f}^{\rho_{1}} h_{1} \\
\vdots \\
L_{f}^{\rho_{p}} h_{p}
\end{array}\right)
$$

and

$$
\Gamma(x, \delta)=\left(\begin{array}{ccc}
L_{G_{1}} L_{f}^{\rho_{1}-1} h_{1} & \cdots & L_{G_{m}} L_{f}^{\rho_{1}-1} h_{1} \\
\vdots & \ddots & \vdots \\
L_{G_{1}} L_{f}^{\rho_{p}-1} h_{p} & \cdots & L_{G_{m}} L_{f}^{\rho_{p}-1} h_{p}
\end{array}\right)
$$

where $H(x, \delta) \in \mathcal{K}^{p \times 1}, \Psi(x, \delta) \in \mathcal{K}^{p \times 1}$ and $\Gamma(x, \delta) \in$ $\mathcal{K}^{p \times m}(\delta]$. And for (6), let denote $\Phi$ the observable space from its outputs:

$$
\Phi=\left\{d h_{1}, \cdots, d L_{f}^{\rho_{1}-1} h_{1}, \cdots, d h_{p}, \cdots, d L_{f}^{\rho_{p}-1} h_{p}\right\}
$$

then we have the following theorem.

Theorem 2. For system (6) with outputs $\left(y_{1}, \ldots, y_{p}\right)$ and corresponding $\left(\rho_{1}, \ldots, \rho_{p}\right)$ with $\rho_{i}=\min \left\{k_{i}, \nu_{i}\right\}$ where $k_{i}$ and $\nu_{i}$ are respectively the associated observability indices and the relative degree, if

$$
\operatorname{rank}_{\mathcal{K}(\delta]} \Phi=n
$$

where $\Phi$ defined in (15), then there exists a change of coordinate $\phi(x, \delta)$ such that (6) can be transformed into (11-13) with $\xi=0$.

Moreover, if the change of coordinate is bicausal over $\mathcal{K}$, then the state $x(t)$ of $(6)$ is causally observable.

For the matrix $\Gamma \in \mathcal{K}^{p \times m}(\delta]$ where $m \leq p$, if

$$
\operatorname{rank}_{\mathcal{K}(\delta]} \Gamma=m
$$

then there exists a matrix $Q \in \mathcal{K}^{p \times p}(\delta]$ such that

$$
Q \Gamma=\left[\begin{array}{l}
\bar{\Gamma} \\
\mathbf{0}
\end{array}\right]
$$

where $\bar{\Gamma} \in \mathcal{K}^{m \times m}(\delta]$ has full row rank $m$. Moreover, if $\bar{\Gamma} \in \mathcal{K}^{m \times m}(\delta]$ is also unimodular over $\mathcal{K}(\delta]$, then the unknown input $u(t)$ of $(6)$ can be causally estimated as well.

Proof 2. According to Theorem 1, (6) can be transformed into (11-13) by $(z, \xi)=\phi(x, \delta)$. Hence, if $\operatorname{rank}_{\mathcal{K}(\delta]} \Phi=n$, where $\Phi$ defined in (15), then one has $\sum_{j=1}^{p} \rho_{j}=n$, which implies (6) can be transformed into (11-13) with $\xi=0$ and the change of coordinate is given by $z=\phi(x, \delta)$ where $z=\left(z_{i}^{T}, \cdots, z_{p}^{T}\right)^{T}$ and $z_{i}=\left(h_{i}, \cdots, L_{f}^{\rho_{i}-1} h_{i}\right)^{T}$.

Moreover, if $\phi(x, \delta) \in \mathcal{K}^{n \times 1}$ is bicausal over $\mathcal{K}$, one can write $x$ as a function of $y_{i}$, its derivative and backward shift, which implies state $x$ is causally observable.

(14) as follows

$$
\Gamma u=H(x, \delta)-\Psi(x, \delta)=\Upsilon(x, \delta)
$$

Since $\operatorname{rank}_{\mathcal{K}(\delta]} \Phi=n$ and $x$ is causally observable, then $\Upsilon(x, \delta)$ is a vector of known meromorphic functions belonging to $\mathcal{K}$. 
According to Lemma 4 in Marquez-Martinez and Moog (2007), for any matrix $\Gamma \in \mathcal{K}^{p \times m}$, if $\operatorname{rank}_{\mathcal{K}(\delta]} \Gamma=m$, by some necessary manipulations, one can always find at least a matrix $Q \in \mathcal{K}^{p \times p}$ such that $Q \Gamma=\left[\begin{array}{l}\bar{\Gamma} \\ \mathbf{0}\end{array}\right]$ where $\bar{\Gamma} \in \mathcal{K}^{m \times m}(\delta]$ has full row rank $m$. Thus, if $\bar{\Gamma} \in \mathcal{K}^{m \times m}(\delta]$ is unimodular over $\mathcal{K}(\delta]$, then there exists a matrix $\bar{\Gamma}^{-1} \in$ $\mathcal{K}^{m \times m}(\delta]$ such that

$$
\left[\begin{array}{ll}
\bar{\Gamma}^{-1} \mathbf{0}
\end{array}\right] Q \Gamma=I_{m \times m}
$$

and

$$
u=\left[\begin{array}{ll}
\bar{\Gamma}^{-1} & \mathbf{0}
\end{array}\right] Q \Upsilon
$$

Since $\bar{\Gamma}^{-1} \in \mathcal{K}^{m \times m}(\delta], Q \in \mathcal{K}^{p \times p}$ and $\Upsilon \in \mathcal{K}^{p \times 1}$, then $u$ of (6) is also causally observable.

Example 2. Consider the following system:

$$
\left\{\begin{array}{l}
\dot{x}_{1}=-\delta x_{1}+x_{2} \\
\dot{x}_{2}=-\delta x_{3}+u_{1} \\
\dot{x}_{3}=\delta x_{1}+\delta u_{1}+u_{2} \\
\dot{x}_{4}=-x_{4}+2 \delta x_{4} / 3 \\
y_{1}=x_{1} \\
y_{2}=x_{3} \\
y_{3}=x_{4}
\end{array}\right.
$$

One can check that $\nu_{1}=k_{1}=2, \nu_{2}=k_{2}=1, \nu_{3}=\infty$ and $k_{3}=1$, then one gets $\rho_{1}=2, \rho_{2}=1$ and $\rho_{3}=1$. According to (15), one has

$$
\begin{aligned}
\Phi & =\left\{d h_{1}, d L_{f} h_{1}, d h_{2}, d h_{3}\right\} \\
& =\left\{d x_{1},-\delta d x_{1}+d x_{2}, d x_{3}, d x_{4}\right\}
\end{aligned}
$$

Since $\operatorname{rank}_{\mathcal{K}(\delta]} \Phi=4$, this gives the following change of coordinate for (17):

$$
z=\phi(x, \delta)=\left(x_{1}, x_{2}-\delta x_{1}, x_{3}, x_{4}\right)^{T}
$$

One can check that the change of coordinate is bicausal over $\mathcal{K}$, since $x$ can be expressed causally by $z$ as follows:

$$
x=\phi^{-1}=\left(\begin{array}{c}
z_{1} \\
\delta z_{1}+z_{2} \\
z_{3} \\
z_{4}
\end{array}\right)
$$

Thus the state of (17) is causally observable and a straightforward computation gives

$$
\left\{\begin{array}{l}
x_{1}(t)=y_{1}(t) \\
x_{2}(t)=y_{1}(t-\tau)+\dot{y}_{1}(t) \\
x_{3}(t)=y_{2}(t) \\
x_{4}(t)=y_{3}(t)
\end{array}\right.
$$

Moreover, one has

$$
\Gamma=\left(\begin{array}{ll}
1 & 0 \\
\delta & 1
\end{array}\right)
$$

which gives $\operatorname{rank}_{\mathcal{K}(\delta]} \Gamma=m=2$. Thus $\Gamma$ is unimodular over $\mathcal{K}(\delta]$, since one can find

$$
\Gamma^{-1}=\left(\begin{array}{cc}
1 & 0 \\
-\delta & 1
\end{array}\right)
$$

such that $\Gamma^{-1} \Gamma=I_{2 \times 2}$. According to Theorem 2, the unknown inputs $u_{1}$ and $u_{2}$ are also causally observable. One can easily obtain the following equations:

$$
\left\{\begin{aligned}
u_{1}(t)= & \dot{y}_{1}(t-\tau)+\ddot{y}_{1}(t)+y_{2}(t-\tau) \\
u_{2}(t)= & \dot{y}_{2}(t)-y_{1}(t-\tau)-\dot{y}_{1}(t-2 \tau) \\
& -\ddot{y}_{1}(t-\tau)-y_{2}(t-2 \tau)
\end{aligned}\right.
$$

Remark 5. For the case where $\operatorname{rank}_{\mathcal{K}(\delta]} \Phi<n$, although Theorem 2 is not valid, it is still possible to estimate the state and the unknown inputs of (6) provided that some complementary conditions are satisfied. In Barbot et al. (2009), a constructive algorithm to solve this problem for nonlinear systems without delays has been proposed, which in fact can be generalized to treat the same problem for nonlinear time-delay systems.

Let us remark that it is the bicausal change of coordinate which makes the state of system causally observable. And it is the unimodular characteristic of $\Gamma$ over $\mathcal{K}(\delta]$ which guarantees the causal reconstruction of unknown inputs. The next section is devoted to dealing with the non-causal case.

\section{NON-CAUSAL OBSERVABILITY}

In order to treat the non-causal case, let introduce the forward time-shift operator $\nabla$, which is similar to the backward time-shift operator $\delta$ defined in Section 2:

$$
\begin{aligned}
\nabla f(t) & =f(t+\tau) \\
\nabla^{i} \delta^{j} f(t) & =\delta^{j} \nabla^{i} f(t)=f(t-(j-i) \tau)
\end{aligned}
$$

for $i, j \in N$

Following the same principle of Section 2 , denote $\overline{\mathcal{K}}$ the field of meromorphic functions of a finite number of variables from $\left\{x_{j}(t-i \tau), j \in[1, n], i \in S\right\}$ where $S=$ $\{-s, \cdots, 0, \cdots, s\}$ is a finite set of constant and one has $\mathcal{K} \subseteq \overline{\mathcal{K}}$. Denote $\mathcal{K}(\delta, \nabla]$ the set of polynomials of the form as follows:

$$
\begin{aligned}
a(\delta, \nabla]= & \bar{a}_{r_{\bar{a}}} \nabla^{r_{\bar{a}}}+\cdots+\bar{a}_{1} \nabla \\
& +a_{0}(t)+a_{1}(t) \delta+\cdots+a_{r_{a}}(t) \delta^{r_{a}}
\end{aligned}
$$

where $a_{i}(t)$ and $\bar{a}_{i}(t)$ belonging to $\overline{\mathcal{K}}$. Let keep the same definition of addition for $\overline{\mathcal{K}}(\delta, \nabla]$, and the multiplication is given as follows:

$$
\begin{aligned}
a(\delta, \nabla] b(\delta, \nabla]= & \sum_{i=0}^{r_{a}} \sum_{j=0}^{r_{b}} a_{i} \delta^{i} b_{j} \delta^{i+j}+\sum_{i=0}^{r_{a}} \sum_{j=1}^{r_{\bar{b}}} a_{i} \delta^{i} \bar{b}_{j} \delta^{i} \nabla^{j} \\
& +\sum_{i=1}^{r_{\bar{a}}} \sum_{j=0}^{r_{b}} \bar{a}_{i} \nabla^{i} b_{j} \nabla^{i} \delta^{j}+\sum_{i=1}^{r_{\bar{a}}} \sum_{j=1}^{r_{\bar{b}}} \bar{a}_{i} \nabla^{i} \bar{b}_{j} \nabla^{i+j}
\end{aligned}
$$

It is clear that $\mathcal{K}(\delta] \subseteq \overline{\mathcal{K}}(\delta, \nabla]$ and the $\operatorname{ring} \overline{\mathcal{K}}(\delta, \nabla]$ possesses the same properties as $\mathcal{K}(\delta]$. Thus a module can be also defined over $\overline{\mathcal{K}}(\delta, \nabla]$ :

$$
\overline{\mathcal{M}}=\operatorname{span}_{\overline{\mathcal{K}}(\delta, \nabla]}\{d \xi, \xi \in \overline{\mathcal{K}}\}
$$

Given the above definitions, Theorem 2 can then be extended as follows in order to deal with non-causal observability for nonlinear time-delay systems.

Theorem 3. For system (6) with outputs $\left(y_{1}, \ldots, y_{p}\right)$ and corresponding $\left(\rho_{1}, \ldots, \rho_{p}\right)$, if

$$
\operatorname{rank}_{\mathcal{K}(\delta]} \Phi=n
$$

where $\Phi$ defined in (15), then there exists a change of coordinate $z=\phi(x, \delta)$ such that (6) can be transformed into (11-13) with $\xi=0$.

Moreover, if the change of coordinate $z=\phi(x, \delta)$ is bicausal over $\overline{\mathcal{K}}$, then the state $x(t)$ of $(6)$ is at least noncausally observable. 
For the deduced matrix $\Gamma$ with $\operatorname{rank}_{\mathcal{K}(\delta]} \Gamma=m$, one can obtain a matrix $\bar{\Gamma} \in \mathcal{K}^{m \times m}(\delta]$ which has full row rank $m$. If $\bar{\Gamma}$ is unimodular over $\overline{\mathcal{K}}(\delta, \nabla]$, then the unknown input $u(t)$ of (6) can be at least non-causally estimated as well.

Proof 3. If the change of coordinate $z=\phi(x, \delta) \in \mathcal{K}^{n \times 1} \subseteq$ $\overline{\mathcal{K}}^{n \times 1}$ is bicausal over $\overline{\mathcal{K}}$, then there exist $\phi^{-1} \in \overline{\mathcal{K}}^{n \times 1}$ and certain $c_{1}, \cdots, c_{n}$ such that

$$
\mathcal{T} x=\phi^{-1}(z, \delta)
$$

where $\mathcal{T}=\operatorname{diag}\left\{\delta^{c_{1}}, \cdots, \delta^{c_{n}}\right\}$. Thus one can define the matrix $\mathcal{T}^{-1}=\operatorname{diag}\left\{\nabla^{c_{1}}, \cdots, \nabla^{c_{n}}\right\} \in \overline{\mathcal{K}}^{n \times n}(\delta, \nabla]$, such that

$$
x=\mathcal{T}^{-1} \phi^{-1}(\delta, z) \in \overline{\mathcal{K}}^{n \times 1}
$$

which means $x$ is at least non-causally observable.

For the estimation of $u(t)$, if $\bar{\Gamma}$ is unimodular over $\overline{\mathcal{K}}(\delta, \nabla]$, then following the same procedure of Theorem 2 , one gets

$$
u=\left[\begin{array}{ll}
\bar{\Gamma}^{-1} \mathbf{0} & Q \Upsilon
\end{array}\right.
$$

In this case, since $\bar{\Gamma}^{-1} \in \overline{\mathcal{K}}(\delta, \nabla], Q \in \mathcal{K}^{p \times p}(\delta]$ and $\Upsilon \in \mathcal{K}^{p \times 1}$, then $u$ of $(6)$ is at least non-causally observable.

Remark 6. Non-causal observations of the state and the unknown inputs are in some case very useful. Because of the non causality, $x$ and $u$ are estimated with some known delays. Many proposed delay feedback control methods can then be applied for stabilizing nonlinear time-delay systems (Sename (2007)). At the other hand, other applications, such as cryptography based on chaotic system, need not the real time estimation, hence non-causal observations can still play an important role in those applications.

\section{CONCLUSION}

This paper introduced a new generic definition of observability for time-delay systems with unknown inputs, covering causal and non-causal observability. Then the relative degree and observability indices for nonlinear time-delay systems were defined based on the notation of the Lie derivation in the framework of non-commutative rings. After that, we introduced a canonical form for time-delay systems, and sufficient conditions were given to guarantee the causal and non-causal observations of states and unknown inputs of time-delay systems.

\section{REFERENCES}

M. Anguelova and B. Wennberga. State elimination and identifiability of the delay parameter for nonlinear timedelay systems. Automatica, 44(5):1373-1378, 2008.

J.-P. Barbot, M. Fliess, and T. Floquet. An algebraic framework for the design of nonlinear observers with unknown inputs. IEEE Conference on Decision and Control, pages 384-389, 2007.

J.-P. Barbot, D. Boutat, and T. Floquet. An observation algorithm for nonlinear systems with unknown inputs. Automatica, 45:1970-1974, 2009.

K. Bhat and H. Koivo. Modal characterizations of controllability and observability in time delay systems. IEEE Transactions on Automatic Control, 21(2):292293, 1976.
M. Boutayeb. Observers design for linear time-delay systems. Systems \& Control Letters, 44(2):103-109, 2001.

J.W. Brewer, J.W. Bunce, and F.S. Van Vleck. Linear systems over commutative rings. Marcel Dekker, New York, 1986.

G. Conte, C.H. Moog, and A.M. Perdon. Nonlinear control systems: An algebraic setting. Lecture Notes in Control and Information Sciences, 242:Springer-Verlag,London, 1999.

M. Darouach. Full order unknown inputs observers design for delay systems. in Proc. of IEEE Mediterranean Conference on Control and Automation, 2006.

S. Diop and M. Fliess. Nonlinear observability, identifiability and persistent trajectories. in Proc. of 36th IEEE Conf. on Decision and Control, 1991.

M. Fliess and H. Mounier. Controllability and observability of linear delay systems: an algebraic approach. ESAIM: Control, Optimisation and Calculus of Variations, 3:301-314, 1998.

T. Floquet and J.-P. Barbot. Super twisting algorithm based step-by-step sliding mode observers for nonlinear systems with unknown inputs. International Journal of Systems Science, 38(10):803-815, 2007.

J.-P. Gauthier, H. Hammouri, and S. Othman. A simple observer for nonlinear systems with applications to bioreactors. IEEE Transactions on Automatic Control, 37(6):875-880, 1992.

A. Germani, C. Manes, and P. Pepe. An asymptotic state observer for a class of nonlinear delay systems. Kybernetika, 37(4):459-478, 2001.

A. Germani, C. Manes, and P. Pepe. A new approach to state observation of nonlinear systems with delayed output. IEEE Transactions on Automatic Control, 47 (1):96-101, 2002.

R. Hermann and A.J. Krener. Nonlinear controllability and observability. IEEE Transactions on Automatic Control, 22(5):728-740, 1977.

S. Ibrir. Adaptive observers for time-delay nonlinear systems in triangular form. Automatica, 45(10):23922399, 2009.

A. Isidori. Nonlinear control systems (3rd edition). London: Springer-Verlag, 1995.

J. Ježek. Rings of skew polynomials in algebraical approach to control theory. Kybernetika, 32(1):63-80, 1996.

A.J. Krener. $\left(\mathrm{A} d_{f, g}\right),\left(a d_{f, g}\right)$ and locally $\left(a d_{f, g}\right)$ invariant and controllability distributions. SIAM Journal on Control and Optimization, 23(4):523-549, 1985.

L.A. Marquez-Martinez and C.H. Moog. New insights on the anlysis of nonlinear time-delay systems: Application to the triangular equivalence. Systems $\&$ Control Letters, 56:133-140, 2007.

L.A. Marquez-Martinez, C.H. Moog, and V.V. Martin. Observability and observers for nonlinear systems with time delays. Kybernetika, 38(4):445-456, 2002.

C.H. Moog, R. Castro-Linares, M. Velasco-Villa, and L. A. Marque-Martinez. The disturbance decoupling problem for time-delay nonlinear systems. IEEE Transactions on Automatic Control, 45(2), 2000.

T. Oguchi and J.-P. Richard. Sliding-mode control of retarded nonlinear systems via finite spectrum assignment approach. IEEE Transactions on Automatic Control, 51 
(9):1527-1531, 2006.

T. Oguchi, A. Watanabe, and T. Nakamizo. Input-output linearization of retarded non-linear systems by using an extension of lie derivative. International Journal of Control, 75(8):582-590, 2002.

P. Pepe. Approximated delayless observers for a class of nonlinear time delay systems. in Proc. of IEEE Conference on Decision and Control, 2001.

K.M. Przyiuski and A. Sosnowski. Remarks on strong observability and detectability of linear time delay systems with disturbances. Systems $\&$ Control Letters, 5(2):121125, 1984.

J.-P. Richard. Time-delay systems: an overview of some recent advances and open problems. Automatica, 39(10): 1667-1694, 2003.

O. Sename. Is a mixed design of observer-controllers for time-delay systems interesting? Asian Journal of Control, 9(2):180-189, 2007.

O. Sename and C. Briat. New trends in design of observers for time-delay systems. Kybernetica, 37(4):427-458, 2001.

O. Sename and C. Briat. $\mathrm{H}_{\infty}$ observer design for uncertain time-delay systems. in Proc. of European Control Conference, 2007.

A. Seuret, T. Floquet, J.-P. Richard, and S.K. Spurgeon. A sliding mode observer for linear systems with unknown time-varying delay. in Proc. of IEEE American Control Conference, 2007.

E. Sontag. A concept of local observability. Systems \& Control Letters, 5:41-47, 1984.

E.D. Sontag. Linear systems over commutative rings: A survey. Ricerche di Automatica, 7:1-34, 1976.

X. Xia, L.A. Marquez, P. Zagalak, and C.H. Moog. Analysis of nonlinear time-delay systems using modules over non-commutative rings. Automatica, 38:1549-1555, 2002.

Y. Xiong and M. Saif. Sliding mode observer for nonlinear uncertain systems. IEEE Transactions on Automatic Control, 46(12):2012-2017, 2001.

J. Zhang, X. Xia, and C.H. Moog. Parameter identifiability of nonlinear systems with time-delay. IEEE Transactions on Automatic Control, 47:371C375, 2006. 